\title{
Morbidity in congenital heart surgery in a public hospital in Argentina
}

\author{
María Althabe, M.D. ${ }^{a}$, Ricardo Rodríguez R., M.D. ${ }^{a}$, María Balestrini, M.D. ${ }^{a}$, \\ Alberto Charroqui, M.D. ${ }^{a}$, Mariela Krynski, M.D. ${ }^{a}$, Ana M. Lenz, M.D. ${ }^{a}$, \\ Mercedes Montonati, M.D. ${ }^{a}$, Guillermo Moreno, M.D. ${ }^{a}$, María L. Pilan, M.D. ${ }^{a}$, \\ Ricardo Magliola, M.D. ${ }^{a}$ and Pablo García Delucis, M.D. ${ }^{b}$
}

\begin{abstract}
Objective: To describe the complications associated with heart surgery, compare them to a reference population, and identify mortality risk factors.

Patients and methods: Retrospective and descriptive study. All patients who underwent surgery at Hospital Garrahan in the 2013-2015 period were included. Age, weight, procedure, mechanical ventilation, length of stay in days, morbidity, and course were recorded. Renal failure requiring dialysis, neurological deficit, permanent pacemaker, circulatory support, phrenic nerve or vocal cord palsy, reoperation, wound infection, chylothorax, and tracheotomy were considered morbidities. A descriptive, statistical analysis by risk category was done using the Society of Thoracic Surgeons (STS) morbidity score.

Results: 1536 patients, median age: 12 months (interquartile range [IQR] 25-75: 3-60), weight: $8 \mathrm{~kg}$ (IQR 25-75:4.4 to 17.5), mortality:5\%. A total of 361 events were recorded in 183 patients. An unplanned reoperation was the most common event $(7.2 \%)$; the rest occurred in $\leq 3 \%$ of patients. Compared to patients without complications, patients who had events required more days on mechanical ventilation: 9.95 (IQR 25-75: 7.6512.24) versus 1.8(IQR 2575: 1.46-2.14), $p<0.00001$; a longer length of stay: 28.8 (IQR 25-75: 25.1-32.5) versus 8.5(IQR25-75:7.9-9.2), $p<0.0001$; and had a higher mortality: $19.6 \%$ versus $3.1 \%$ (RR: 4.58 , 95\% CI:3.4 to 6.0), $p<0.0001$. Circulatory support and renal failure were associated with a higher mortality.

Conclusions: An unplanned reoperation was the most common event. Patients with complications required more days on mechanical ventilation and a longer length of stay and had a higher mortality. Circulatory support and renal failure were associated with a higher mortality. Key words: morbidity, congenital heart diseases, cardiovascular surgical procedures.
\end{abstract}

http:/ / dx.doi.org/10.5546/ aap.2018.eng.e14

María Althabe, M.D.: maria.althabe@gmail.com

Funding:

None.

Conflict of interest:

None.

Received: 4-3-2017

Accepted: 8-9-2017

To cite: Althabe M, Rodríguez RR, Balestrini et al. Morbidity in congenital heart surgery in a public hospital in Argentina. Arch Argent Pediatr 2018;116(1):e14-e18.

\section{INTRODUCTION}

Given the sustained decrease in postoperative mortality rates, the reduction in morbidity in the setting of congenital heart surgery is one of the main pillars of the improved quality initiatives for the management of these patients. Postoperative complications following a pediatric cardiovascular surgery are very common; different publications regarding reference centers have described values above $30 \% .{ }^{1}$ Complications not only extend the length of stay and increase health care costs, many of them also significantly increase the mortality risk. ${ }^{1-3}$ The difference with mortality observed at centers of excellence is not attributed to a lower number of complications but to an improved diagnosis and management of complications..$^{4-6}$ For this reason, in recent years, several instruments have been developed to measure morbidity and establish reference standards to make comparisons and assess performance at different sites. ${ }^{7-9}$ In Argentina, there are no data available on the prevalence of complications in this population. Available local results would mean an advance in outcome quality.

The objective of this study was to describe the complications associated with congenital heart surgery, compare them to a reference population, and identify those that are risk factors for mortality in our population.

\section{PATIENTS AND METHODS}

This was a retrospective and descriptive study. All patients who underwent a congenital heart 
surgery and recovered in the Cardiovascular Intensive Care Unit of Hospital Garrahan between $1 / 1 / 2013$ and $12 / 31 / 2015$ were included if their procedure was allocated a risk category according to the Risk Adjustment For Congenital Heart Surgery (RACHS-1), a scale made up of six categories of increasing mortality risk depending on the complexity of the procedure, e.g., a ductus is allocated category 1 whereas the Norwood procedure is category $6 .{ }^{10}$ Hospital de Pediatría "Dr. J. P. Garrahan" is a tertiary care referral facility in this region, where approximately 600 patients with congenital heart disease are operated on each year. It has a Cardiovascular Intensive Care Unit for recovery with 21 beds, and the postoperative mortality rate here in recent years has been $5 \% .11,12$

Age, weight, surgery type, days on mechanical ventilation (MV), length of stay in the Intensive Care Unit (ICU) in days, morbidity, and course were recorded as outcome measures. Morbidity was defined as the presence of at least one of the following events: acute renal failure requiring dialysis, neurological deficit persistent at discharge, third-degree atrioventricular block requiring permanent pacemaker, mechanical circulatory support, phrenic nerve palsy, unplanned reoperation, vocal cord palsy, surgical wound infection, chylothorax, and tracheotomy requirement in the 30 days following surgery. The first six events were defined as major complications according to the Society of Thoracic Surgeons Congenital Heart Surgery Database (STSCHSD), which includes registries from 62

TABLE 1. Description of the population ( $n$ : 1536)

\begin{tabular}{lcc}
\hline Outcome measure & & \\
\hline Age (months) Median and IQR 25-75 & $12(3-60)$ & \\
Male sex & $53 \%$ & \\
Weight (kg) Median and IQR 25-75 & $8(4.4-17.5)$ & \\
RACHS-1 category $\left(^{*}\right)$ & & Mortality \\
R 1 & $14 \%$ & $0 \%$ \\
R2 & $37 \%$ & $1.94 \%$ \\
R3 & $36 \%$ & $6.67 \%$ \\
R4 & $9.9 \%$ & $12.5 \%$ \\
R5/6 & $3.1 \%$ & $22.9 \%$ \\
Genetic disorder & $14 \%$ & \\
Other malformations & $4.5 \%$ & \\
Other diseases & $4.6 \%$ & \\
Preoperative MV & $10.1 \%$ & \\
Prior surgery & $26.8 \%$ & \\
\hline
\end{tabular}

IQR: interquartile range; MV: mechanical ventilation; RACHS-1: Risk Adjustment for Congenital Heart Surgery.
851 surgeries. The Society of Thoracic Surgeons (STS) morbidity score was developed based on the analysis of frequency of these events for the different procedures. It identified five risk categories of increasing morbidity risk stratified depending on the complexity of the surgery: category 1 for minor risk procedures and 5 for major risk ones. ${ }^{8}$ The four remaining events were selected by the authors because of their potential impact on mortality, length of stay, and special home care requirements.

The protocol was approved by the Research Review Committee. Data were collected from the department's database using prospective records and were dissociated to maintain confidentiality. A statistical, descriptive analysis was done; results were described as median and interquartile range (IQR 25-75) or as absolute value and percentage, as applicable. To make a comparison with data published for a reference population, the $\chi^{2}$ test was used for discrete outcome measures and the Wilcoxon rank-sum test was used for continuous outcome measures; a $p$ value $<0.05$ was considered significant ${ }^{8}$ (Stata 10).

\section{RESULTS}

A total of 1536 patients were included in the study period. Population data are described in Table 1. Among all patients, $10 \%$ were newborn infants and $8.7 \%$ weighted $\leq 3 \mathrm{~kg}$. The overall mortality rate was 5\%. A total of 361 events were recorded in 183 patients; their frequency distribution is shown in Table 2. An unplanned reoperation was the most common event $(7.2 \%)$.
TABLE 2. Complications in order of frequency ( $n: 361)$

\begin{tabular}{lcc}
\hline Complications & $\mathbf{n}$ & $\%$ (**) $^{*}$ \\
\hline Unplanned reoperation $\left(^{*}\right)$ & 110 & 7.2 \\
Acute renal failure requiring dialysis $\left(^{*}\right)$ & 47 & 3.1 \\
Surgical wound infection & 46 & 3.0 \\
Phrenic nerve palsy $\left(^{*}\right)$ & 36 & 2.3 \\
Mechanical circulatory support $\left(^{*}\right)$ & 28 & 1.8 \\
Chylothorax & 23 & 1.5 \\
Neurological deficit persistent & & \\
at discharge $\left(^{*}\right)$ & 21 & 1.4 \\
Vocal cord palsy & 19 & 1.2 \\
Third-degree atrioventricular block & & \\
requiring permanent pacemaker $\left(^{*}\right)$ & 16 & 1.0 \\
Tracheotomy & 15 & 1.0 \\
Total & 361 & \\
\hline
\end{tabular}

$\left.{ }^{*}\right)$ Events included in the morbidity score.

$\left.{ }^{(* *}\right)$ Frequency of each event out of all surgeries. 
Patients with events required more days on MV: 9.95 (IQR 25-75: 7.65-12.24) versus 1.8 (IQR 2575: 1.46-2.14), $p<0.00001$; a longer length of stay: 28.8 (IQR 25-75: 25.1-32.5) versus 8.5 (IQR 25-75: 7.9-9.2), $p<0.0001$; and had a higher mortality: $19.6 \%$ versus $3.1 \%$ (RR: $4.58,95 \%$ CI: 3.4 to 6.0 ), $p<0.0001$. Such difference was maintained regardless of the RACHS-1 category to which they were allocated.

Comparative analysis: 1533 patients were allocated a category according to the STS morbidity score; $45 \%$ were category $1 ; 17 \%$, category $2 ; 20 \%$, category $3 ; 15.7 \%$, category 4 ; and $2.1 \%$, category 5 . Table 3 describes the event frequency by category. Most events had an increasing frequency in accordance with their risk category, except for the neurological complication and atrioventricular block requiring permanent pacemaker. Morbidity by category and its comparison to international results are shown in Table 4. The proportion of events in our population is significantly higher in all categories, except for category 5 , which encompasses more complex procedures. Table 5 compares the frequency of each event and Table 6, mortality associated with each event.

\section{DISCUSSION}

Initiatives to improve health care quality in children with congenital heart disease have multiplied in recent years aimed at reducing mortality and morbidity in this group of patients. The harmonization of diagnosis classification, surgical procedures, and complications has helped to mainstream the results of most pediatric heart surgery centers. The unified STSCHSD has

TABLE 3. Frequency of events according to the Society of Thoracic Surgeons morbidity score categories

\begin{tabular}{lcccccccc}
\hline Category & $\mathbf{n}$ & $\begin{array}{c}\mathbf{D} \\
\boldsymbol{\%}\end{array}$ & $\begin{array}{c}\mathbf{N} \\
\mathbf{( \% )}\end{array}$ & $\begin{array}{c}\text { PPM } \\
\%\end{array}$ & $\begin{array}{c}\text { ECMO } \\
\%\end{array}$ & $\begin{array}{c}\text { PNP } \\
\%\end{array}$ & $\begin{array}{c}\text { UR } \\
\%\end{array}$ & $\begin{array}{c}\mathbf{Q} \\
\%\end{array}$ \\
\hline 1 & 689 & 0.1 & 1 & 1 & 0.6 & 0.6 & 3.8 & 3.2 \\
2 & 264 & 0.8 & 0.4 & 0.4 & 1.9 & 3.8 & 9.5 & 6.4 \\
3 & 308 & 5.8 & 2.6 & 1.9 & 2.9 & 3.2 & 9.7 & 13.6 \\
4 & 240 & 7.9 & 0.8 & 0.8 & 3.8 & 3.8 & 9.6 & 17.5 \\
5 & 32 & 21.9 & 9.4 & 0 & 3.1 & 9.4 & 18.8 & 34.4 \\
Total & 1533 & & & & & & & \\
\hline
\end{tabular}

D: dialysis; N: neurological deficit persistent at discharge; PPM: permanent pacemaker; ECMO: extracorporeal membrane oxygenation circulatory support; PNP: phrenic nerve palsy; UR: unplanned reoperation; Q: patients with 1 or more complications.

TABLE 4. Morbidity based on the Society of Thoracic Surgeons morbidity score. Comparison to the reference population

\begin{tabular}{|c|c|c|c|c|c|c|}
\hline \multirow[b]{2}{*}{ Categoría } & \multicolumn{2}{|c|}{ Garrahan } & \multicolumn{2}{|c|}{ Reference $\left({ }^{*}\right)$} & \multirow[b]{2}{*}{$\operatorname{RR}(95 \% \mathrm{CI})$} & \multirow[b]{2}{*}{$p$} \\
\hline & $\mathbf{n}$ & $\%$ & $\%$ & $\%$ & & \\
\hline 1 & 689 & 44.9 & 7.1 & 3.2 & $2.2(1.68-2.94)$ & 0.000000 \\
\hline 2 & 264 & 17.2 & 16.7 & 6.5 & $2.56(1.95-3.38)$ & 0.000000 \\
\hline 3 & 308 & 20.1 & 26.3 & 11.9 & $2.21(1.82-2.69)$ & 0.000000 \\
\hline 4 & 240 & 15.7 & 26.7 & 15.2 & $1.75(1.41-2.18)$ & 0.000001 \\
\hline 5 & 32 & 2.1 & 62.5 & 30.0 & $1.28(0.90-1.82)$ & 0.18 \\
\hline Total & 1533 & & & & & \\
\hline
\end{tabular}

${ }^{*}$ ) Based on 62851 surgeries. Society of Thoracic Surgeons Congenital Heart Surgery Database. ${ }^{8}$

RR: relative risk; CI: confidence interval.

TABLE 5. Comparison of frequency by event type to the reference population

\begin{tabular}{lcccccc}
\hline & Reference (n: 62 851) & \multicolumn{2}{c}{ Garrahan (n:1533) } & & \\
Events & $\mathbf{n}$ & $\%$ & $\mathbf{~ n}$ & $\%$ & RR (95\% CI) & $\boldsymbol{p}$ \\
\hline Acute renal failure requiring dialysis & 705 & 1.1 & 47 & 3.1 & $2.7(2.0-3.6)$ & 0.000000 \\
Neurological deficit persisting at discharge & 500 & 0.8 & 21 & 1.4 & $1.7(1.1-2.6)$ & 0.019 \\
Atrioventricular block requiring permanent pacemaker & 593 & 0.9 & 16 & 1 & $1.1(0.6-1.8)$ & 0.78 \\
Mechanical circulatory support & 1110 & 1.8 & 28 & 1.8 & $1.03(0.7-1.49)$ & 0.93 \\
Phrenic nerve palsy & 578 & 0.9 & 36 & 2.3 & $2.55(1.8-3.6)$ & 0.000000 \\
Unplanned reoperation & 2942 & 4.7 & 110 & 7.2 & $1.5(1.2-1.8)$ & 0.000007 \\
Patients with 1 or more complications & 5059 & 8.0 & 127 & 8.3 & $1.1(0.8-1.2)$ & 0.77 \\
\hline
\end{tabular}

RR: relative risk; CI: confidence interval. 
registered data for 479000 procedures, since 1998 to date. ${ }^{13}$ Among other things, this type of initiative serves to assess the impact of new practices and strategies in a shorter period of time given the large number of analyzable patients. In 2013, based on these data, an instrument was proposed to measure morbidity based on the empirical observation of results of 62851 surgeries in 68 centers, and this is the tool used in our study. ${ }^{8}$

To our knowledge, this was the first study that analyzed in detail morbidities associated with congenital heart surgery in Argentina. For this reason, it was not possible to compare our results to those of local or regional centers. The number of events in our population was higher than in the reference population; however, the rate of mortality was according to international standards. The factors that impact on the performance of the centers analyzed in the bibliography include the volume of procedures carried out, patients' preoperative condition and comorbidities. ${ }^{3,5,6}$ Our population covers a high percentage of patients with associated genetic disorders and prior surgeries, and many are on MV at the time of surgery. The fact that this is a teaching facility with a high human resource turnover is a factor that deserves analysis but our study was not aimed at assessing this hypothesis. The association between morbidity and mortality has been identified in multiple studies conducted in children and adults, and was confirmed by our results. The mortality rate among patients with major complications was six times higher than among those without major events. The probability of dying from a complication or adverse event is known as "rescue failure" and has been proposed as a helpful quality indicator to assess centers' performance. A complication is defined as an event associated with a disease or health intervention; it is an alteration of the desired course of a disease and may cause or be associated with a suboptimal outcome. A complication not necessarily means a gap in the standard of care that accounts for negligence or malpractice. By definition, the centers with a better performance do not have fewer complications but are capable of identifying and managing them in an early and improved manner, thus reducing mortality. ${ }^{5,14,15}$

A limitation of this study was that it did not analyze all potential complications. International databases identify at least 34 complications grouped into different categories: cardiac, pulmonary, renal, neurological, infectious, and surgical complications. The events included in our analysis were selected considering the possibility of comparing them to international references by risk category, which led us to include the events considered in the morbidity score. In relation to the other recorded events, and because this is a retrospective study, the limitation in data collection persuaded us to select few but striking events to minimize any potential underrecording. In spite of its limitations, we believe that this study offers important information for the management of patients with complex heart malformations using an instrument to measure and compare centers' performance. Future prospective studies are necessary to analyze the entire range of complications, not only surgeryrelated ones, but also those associated with diagnosis, transfer, and preoperative stabilization so that objective data becomes available to improve the outcomes of complex congenital heart diseases.

\section{CONCLUSIONS}

An unplanned reoperation was the most common event. Patients with complications required more days on MV and a longer length

TABLE 6. Comparison of mortality by event with the international reference population

\begin{tabular}{lcccc}
\hline & \multicolumn{2}{c}{ Mortality (\%) } & & \\
Postoperative event & Garrahan & Reference & RR (95\% CI) & $p$ \\
\hline Acute renal failure requiring dialysis & 42.6 & 56.2 & $1.30(1.01-1.69)$ & 0.095 \\
Neurological deficit persisting at discharge & 23.8 & 30.4 & $1.09(0.8-1.4)$ & 0.68 \\
Third-degree atrioventricular block requiring permanent pacemake & 0.0 & 4.7 & $1.04(1.03-1.06)$ & 0.77 \\
Mechanical circulatory support & 25.0 & 55.6 & $1.68(1.3-2.1)$ & 0.002 \\
Phrenic nerve palsy & 2.8 & 6.1 & $1.03(0.97-1.09)$ & 0.655 \\
Unplanned reoperation & 13.6 & 21.6 & $1.10(1.0-1.18)$ & 0.06 \\
Patients with 1 or more complications & 25.2 & 23.5 & $0.77(0.7-0.8)$ & 0.000000 \\
\hline
\end{tabular}

Reference: reference population. ${ }^{(8)}$

$\mathrm{RR}$ : relative risk; CI: confidence interval. 
of stay, and had a higher mortality. Mechanical circulatory support and renal failure requiring dialysis were the complications associated with a higher mortality.

\section{REFERENCES}

1. Benavidez OJ, GauvreauK, DelNidoP, et al. Complications and risk factors for mortality during congenital heart surgery admissions. Ann Thorac Surg 2007;84(1):147-55.

2. Agarwal HS, Wolfram KB, Saville BR, et al. Postoperative complications and association with outcomes in pediatric cardiac surgery. J Thorac Cardiovasc Surg 2014;148(2):60916.e1.

3. Bacha EA, Cooper D, Thiagarajan R, et al. Cardiac complications associated with the treatment of patients with congenital cardiac disease: consensus definitions from the Multi-Societal Database Committee for Pediatric and Congenital Heart Disease. Cardiol Young 2008;18(Suppl 2):196-201.

4. Ahmed EO, Butler R, Novick RJ. Failure-to-rescue rate as a measure of quality of care in a cardiac surgery recovery unit: A five-year study. Ann Thorac Surg 2014;97(1):147-52.

5. Pasquali SK, Li JS, Burstein DS, et al. The association of center volume with mortality and complications in pediatric heart surgery. Pediatrics 2012;129(2):e370-6.

6. Pasquali SK, He X, Jacobs JP, et al. Evaluation of failure to rescue as a quality metric in pediatric heart surgery: An analysis of the STS congenital heart surgery database. Ann Thorac Surg 2012;94(2):573-9.

7. Bojan M, Gerelli S, Gioanni S, et al. Evaluation of a new tool for morbidity assessment in congenital cardiac surgery. Ann Thorac Surg 2011;92(6):2200-4.

8. Jacobs ML, O'BrienSM, JacobsJP, etal. An empirically based tool for analyzing morbidity associated with operations for congenital heart disease. J Thorac Cardiovasc Surg 2013; 145(4):1046-57.

9. Lacour-Gayet F, Jacobs ML, Jacobs JP, et al. The Need for an Objective Evaluation of Morbidity in Congenital Heart Surgery. Ann Thorac Surg 2007;84(1):1-2.

10. Jenkins KJ. Risk adjustment for congenital heart surgery: the RACHS-1 method. Semin Thorac Cardiovasc Surg Pediatr Card Surg Annu 2004;7(1):180-4.

11. Magliola R, Althabe M, Moreno G, et al. Cirugía cardiaca reparadora en recién nacidos. Experiencia de 5 años en cirugía neonatal con circulación extracorpórea. Arch Argent Pediatr 2009;107(5):417-22.

12. Magliola RH, Althabe M, Moreno G, et al. Cardiopatías congénitas: Resultados quirúrgicos en un hospital público en Argentina. Arch Cardiol Mex 2011;81(3):178-82.

13. JacobsJP,MayerJE, MavroudisC, etal. TheSociety of Thoracic Surgeons Congenital Heart Surgery Database: 2016 Update on Outcomes and Quality. Ann Thorac Surg 2016;101(3):85062.

14. Burki S, Fraser CD Jr. Larger Centers May Produce Better Outcomes: Is Regionalization in Congenital Heart Surgery a Superior Model? Semin Thorac Cardiovasc Surg Pediatr Card Surg Апnи 2016;19(1):10-3.

15. Danton MHD. Larger Centers Produce Better Outcomes in Pediatric Cardiac Surgery: Regionalization is a Superior Model - The Con Prospective. Semin Thorac Cardiovasc Surg Pediatr Card Surg Annu 2016;19(1):14-24. 